\title{
Precise determination of the sigma pole location from a dispersive analysis
}

\author{
R. García-Martín*, R. Kamiński† and J. R. Peláez* \\ ${ }^{*}$ Dpto. Física Teórica II, Facultad de CC. Físicas, Universidad Complutense, Madrid, Spain. \\ ${ }^{\dagger}$ Institute of Nuclear Physics, Polish Academy of Science, Kraków, Poland
}

\begin{abstract}
We review how the use of recent precise data on kaon decays together with forward dispersion relations (FDR) and Roy's equations allow us to determine the sigma resonance pole position very precisely, by using only experimental input. In addition, we present preliminary results for a modified set of Roy-like equations with only one subtraction, that show a remarkable improvement in the precision around the $\sigma$ region. For practical applications, these results are shown to be very well approximated by a very simple conformal expansion.
\end{abstract}

Keywords: Roy's equations, dispersion relations, sigma, scalar mesons, meson-meson scattering PACS: 11.55.Fv,11.80.Et,13.75.Lb,14.40.Cs

\section{INTRODUCTION}

The values quoted in the Particle Data Table for the sigma mass and width, based on both pole position and Breit-Wigner parameter determinations are very widely spread, with an estimated mass and half width of [1]:

$$
\sqrt{s_{\sigma}} \equiv M_{\sigma}-i \Gamma_{\sigma} / 2 \simeq(400-1200)-i(250-500)(\mathrm{MeV}) .
$$

This large uncertainty is mainly due to the fact that old data sets for pion-pion scattering are poor and often contradictory. Moreover, the choice of data sets varies among different works. To make things worse, there is quite a variety of different ways to extrapolate the data on the real axis to the complex plane, and the pole position of the sigma is greatly affected by model dependences.

This said, model independent techniques for extrapolating amplitudes from the real axis onto the complex plane exist in the form of dispersion relations, which allow us to analytically continue an amplitude away from the real axis provided we know its imaginary part for physical values of the energy. These dispersive techniques have already been successfully used for predicting the position of the sigma pole, with a remarkable agreement among the different works:

$$
\begin{array}{cl}
440-i 245 \mathrm{MeV} & \text { Dobado, Pelaez (1997) [2] } \\
470 \pm 50-i 260 \pm 25 \mathrm{MeV} & \text { Zhou et al. (2005) [3] }
\end{array}
$$

In particular, there exists a dispersive representation that incorporates crossing exactly, written by Roy [4], which involves only the partial wave amplitudes. Roy's equations have already been used to predict the position of the sigma pole from the theoretical 
predictions of ChPT [5], obtaining:

$$
\sqrt{s_{\sigma}}=441_{-8}^{+16}-i 272_{-19.5}^{+9}
$$

In addition, the data coming from the E865 collaboration at Brookhaven [6], and especially the recently published data from NA48/2 [7] provide us with very precise data on pion-pion scattering at very low energies, These allow us to obtain very reliable parametrizations of the S0 wave at low energy [8], from which the scattering lengths can be directly extracted [9] with a remarkable precision and in good agreement with the theoretical predictions of ChPT [5].

Our aim is thus to perform a dispersive analysis, including all available experimental data, in order to give a precise and model independent determination of the sigma pole position, by using exclusively data, analiticity and crossing symmetry. We use both Forward Dispersion Relations (FDR) and Roy's equations, without assuming ChPT, so that we can actually test its predictions.

\section{APPROACH AND RESULTS}

The details on the parametrizations used for the data have been explained fully in this conference (see talk by J. R. Peláez [8]), as well as in Ref. [10] - that we will denote by KPY08. It is enough to say here that two different sets of parameters are considered:

- Unconstrained Fits to Data (UFD), in which each partial wave is fitted independently. This set satisfies both FDR and Roy's equations within the experimental errors in all waves except the Roy equation for the S2 wave, for which the deviation is about $1.3 \sigma$, and the antisymmetric FDR above $930 \mathrm{MeV}$ by a couple of standard deviations.

- Constrained Fits to Data (CFD), obtained by constraining the fits to satisfy simultaneously FDR and Roy's equations, so that all waves are correlated. The CFD set provides a remarkably precise and reliable description of the experimental data, and at the same time satisfy the analytic properties remarkably well.

These two sets provide a reliable parametrization for the imaginary part of the partial waves that we need as input for Roy's equations.

An elastic resonance has an associated pole on the second Riemann sheet of the complex plane S-matrix, which, as it is well known, corresponds by unitarity to a zero on the first sheet. As usual then, we just need to look numerically for zeroes of the S-matrix on the physical sheet:

$$
S_{0}^{0}(s)=1+2 i \sigma(s) t_{0}^{0}(s),
$$

where the analytic extension of the partial wave amplitudes away from the real axis is given by Roy's equations:

$$
t_{0}^{0}(s)=a_{0}^{0}+\frac{s-4 M_{\pi}^{2}}{12 M_{\pi}^{2}}\left(2 a_{0}^{0}-5 a_{0}^{2}\right)+\sum_{I=0}^{2} \sum_{\ell=0}^{1} \int_{4 M_{\pi}^{2}}^{\Lambda} d s^{\prime} k_{0 \ell}^{0 I}\left(s, s^{\prime}\right) \operatorname{Im} t_{\ell}^{I}(s)+d_{0}^{0}(s) .
$$

The domain of validity of Roy's equations has been shown to cover the region of the complex plane where the sigma lies [5]. 
Taking the UFD set as the input for Roy's equations, we find an S-matrix zero at $\sqrt{s}=(426 \pm 25)-i(241 \pm 17) \mathrm{MeV}$. However, Roy's equations are not completely satisfied by this data set, thus the pole position will be much more reliable if the input satisfies the equations, as it is the case for the CFD set. In this case we find:

$$
\sqrt{s_{\sigma}}=(456 \pm 36)-i(256 \pm 17) \mathrm{MeV},
$$

which still has big uncertainties due to the strong dependence of Roy's equations on the scattering lengths, in particular of the $a_{0}^{2}$, which is known with less precision. These values are, however, subject to further improvement and should be considered preliminary. It should also be noted that they are in perfect agreement with the theoretical prediction by Caprini et al. of $\sqrt{s_{\sigma}}=441_{-8}^{+16}-i 272_{-19.5}^{+9}$.

\section{WORK IN PROGRESS}

The three authors of this work together with F. J. Ynduráin (see J. R. Peláez talk in this conference [8]) have derived a modified set of Roy-like equations which are based on once-subtracted dispersion relations - Roy's equations are twice subtracted. These new equations (GKPY for brevity) have a very different dependence on the observables. In particular, given the same input, the uncertainty dependence on scattering lengths is much weaker. Indeed, at low energy (below $\sim 350 \mathrm{MeV}$ ) we find the uncertainties for GKPY equations bigger than the ones given by Roy's equations, but above $\sim 400 \mathrm{MeV}$ they are already smaller, as they do not increase with energy. This allows us to obtain the position of the sigma pole from Constrained Fits to Data with higher accuracy than using standard Roy's equations alone.

Moreover, we have already performed a preliminary Constrained Fit to Data (CFD-II) in which these new equations are also imposed as new constraints within errors (see [8]). This gives rise to a new set of parameters which better encode the experimental information together with unitarity, analiticity and crossing symmetry, therefore allowing us to obtain a more precise and reliable determination of the sigma pole. The result for the sigma pole position using the very preliminary CFD-II set is:

$$
\begin{aligned}
& \sqrt{s_{\sigma}}=(462 \pm 51)-i(264 \pm 20) \mathrm{MeV}, \text { (preliminary from Roy Eqs.) } \\
& \sqrt{s_{\sigma}}=(458 \pm 15)-i(262 \pm 15) \mathrm{MeV}, \text { (preliminary from GKPY) }
\end{aligned}
$$

although, as it can be seen in the Figure 1a, more work is needed on the $f_{0}(980)$ region, as GKPY equations can discern among solutions that were equivalent for Roy's equations. The analysis should be complete within the next few months.

\section{THE CONFORMAL EXPANSION}

The most rigurous way to extrapolate to the complex plane is by using Roy's or GKPY equations. However, dealing with the whole set of equations is complicated and computationaly tedious. For simple applications, there exists a simple approximate solution, which is very easy to handle: the conformal expansion. This is a model independent 

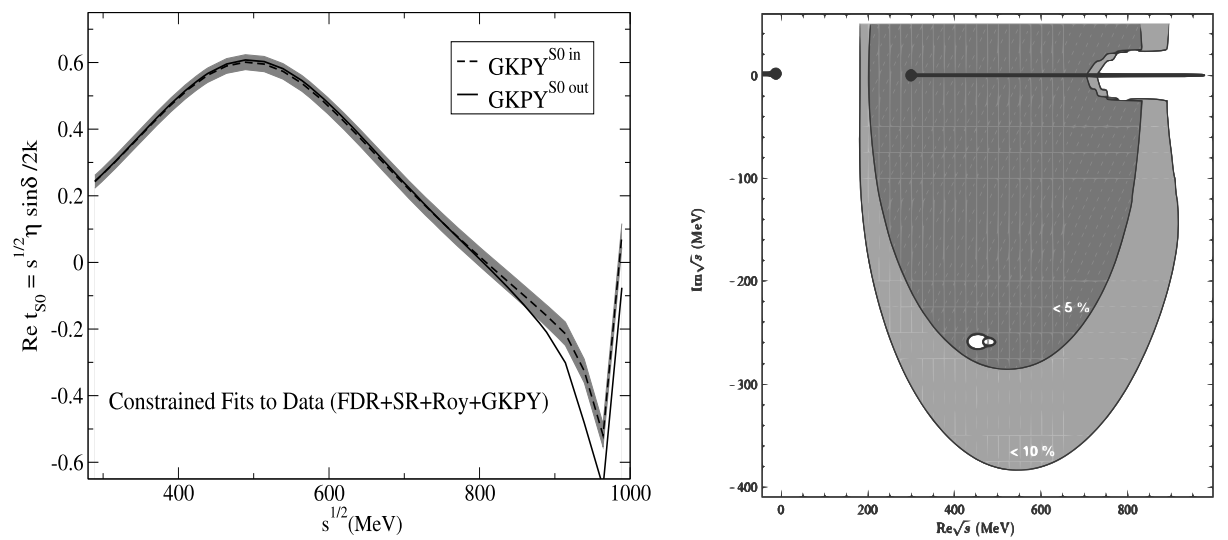

FIGURE 1. a) Difference between input and output of GKPY equations for Re $t_{0}^{0}$, with the corresponding error band. The smaller uncertainties carried by these new equations will allow us to improve the fit in the $f_{0}(980)$ region. b) Relative difference between the conformal expansion and Roy's equations. The contours show the regions inside of which the difference is less than 5 and 10 percent. The sigma pole positions with their errors are plotted as ellipses.

parametrization of the experimental data at low energies, based on unitarity and elasticity, and can describe experimental data with few parameters. The explicit details are explained in full length in Ref. [9], here it suffices to remember that, for elastic scattering, a given partial amplitude of definite isospin $I$ and angular momentum $\ell$ can be written as $t_{\ell}^{I}(s)=\frac{1}{\psi(s)-i \sigma(s)}$, with $\psi(s)$ being the effective range function, which can be series expanded in the conformal variable $\omega(s)=\frac{\sqrt{s}-\sqrt{s_{0}-s}}{\sqrt{s}+\sqrt{s_{0}-s}}$ as follows:

$$
\psi(s)=\frac{M_{\pi}^{2}}{s-z_{0}^{2} / 2}\left\{\frac{z_{0}^{2}}{M_{\pi} \sqrt{s}}+B_{0}+B_{1} \omega(s)+B_{2} \omega(s)^{2}+\ldots\right\}
$$

Three parameters are enough to describe the experimental data below the $\pi \pi \rightarrow \bar{K} K$ inelastic threshold in all studied $\pi \pi$ partial waves $(S, P, D, F$ and $G)$.

We show in Figure $1 \mathrm{~b}$ the complex plane for $\sqrt{s}$, plotting the contours inside of which the relative difference between the amplitude calculated with GKPY equations and calculated with the conformal expansion, i.e., $\Delta_{00}(s)=\frac{\left|t_{00}^{\text {conf }}(s)-t_{00}^{G K P Y}(s)\right|}{\frac{1}{2}\left|t_{00}^{\text {conf }}(s)+t_{00}^{G K P Y}(s)\right|}$, is less than 5 or 10 percent. Note that we use our preliminary parameters obtained by constraining the data fit to satisfy FDR, Roy's equations and GKPY equations. We can see that for the sigma region, the difference between both calculations is less than 5 percent, and indeed the pole locations overlap within their errors: using the same input as for Eq. (8) 
we find:

$$
\sqrt{s_{\sigma}^{\text {conf }}}=(478 \pm 17)-i(262 \pm 7) \mathrm{MeV} .
$$

We see that the systematic uncertainties associated with the conformal expansion, which correspond to neglecting crossing symmetry and the inelastic cut- $f_{0}(980)$ regionare bigger than our previous estimation in Ref. [9], but still within the 5 percent band. The crudeness of our estimation of systematic uncertainties has been pointed out by I. Caprini in this conference [11]. Certainly in ref. [9] we only estimated crudely the systematic error from different parametrizations and truncations of our conformal expansion, and we came up with $\Delta_{\text {sys }} M_{\sigma}= \pm 11 \mathrm{MeV}$ and $\Delta_{\text {sys }} \Gamma_{\sigma} / 2= \pm 2 \mathrm{MeV}$. I. Caprini [11], using an arbitrary sampling of conformal parametrizations and conformal variables - which are different from ours - has provided a new estimate $\Delta_{\text {sys }} M_{\sigma} \simeq 40 \mathrm{MeV}$ and $\Delta_{\text {sys }} \Gamma_{\sigma} / 2= \pm 40 \mathrm{MeV}$, namely a factor of 3 or 4 larger than ours. Nevertheless, the difference between central values for the sigma pole position obtained from GKPY equations and our conformal expansion provides the systematic theoretical uncertainties in our conformal expansion calculation, without depending on a sampling of conformal variables and parametrizations. In view of the differences between Eqs.(9) and (11), we certainly agree that the systematic uncertainty of the pole obtained from our conformal parametrization was underestimated, although not as much as it is suggested in [11] but just by a factor of two $\Delta_{s y s} M_{\sigma} \simeq 20 \mathrm{MeV}$.

To summarize this section, up to the sigma region of the complex plane, our conformal expansion provides a very simple and reliable approximation, accurate to $5 \%$, to the $\pi \pi$ scattering amplitude as obtained from data fits constrained to satisfy FDR, Roy and GKPY equations.

\section{ACKNOWLEDGMENTS}

We thank Prof. F. J. Ynduráin for his comments and suggestions, the organizers for creating the nice scientific atmosphere of the workshop and the Spanish research contracts PR27/05-13955-BSCH, FPA2004-02602, UCM-CAM 910309 and BFM2003-00856 for partial financial support.

\section{REFERENCES}

1. W. M. Yao, et al., J. Phys. G33, 1-1232 (2006).

2. A. Dobado, and J. R. Pelaez, Phys. Rev. D56, 3057-3073 (1997), hep-ph / 9604416.

3. Z. Y. Zhou, et al., JHEP 02, 043 (2005), hep-ph/ 0406271.

4. S. M. Roy, Phys. Lett. B36, 353 (1971).

5. I. Caprini, G. Colangelo, and H. Leutwyler, Phys. Rev. Lett. 96, 132001 (2006), hep-ph / 0512364.

6. S. Pislak, et al., Phys. Rev. Lett. 87, 221801 (2001), hep-ex/ 0106071.

7. J. R. Batley, et al. (2008), cERN-PH-EP-2007-035.

8. J. R. Pelaez, On this conference (2008).

9. F. J. Yndurain, R. Garcia-Martin, and J. R. Pelaez, Phys. Rev. D76, 074034 (2007), hep-ph/ 0701025.

10. R. Kaminski, J. R. Pelaez, and F. J. Yndurain (2008), arXiv: 0710.1150 [hep-ph] .

11. I. Caprini (2008), 0804.2108. 
Copyright of AIP Conference Proceedings is the property of American Institute of Physics and its content may not be copied or emailed to multiple sites or posted to a listserv without the copyright holder's express written permission. However, users may print, download, or email articles for individual use. 